\title{
Classical Derivation of Auxiliary Lorentz Transforms: Their Relations with Special Relativity
}

\author{
Sankar Hajra \\ Indian Physical Society, Calcutta, India \\ Email: sankarhajra@yahoo.com
}

Received July 2, 2012; revised September 2, 2012; accepted September 8, 2012

\begin{abstract}
In this paper we have given a direct deduction of the auxiliary Lorentz transforms from the consideration of Maxwell. In the Maxwell's theory, if $c$ is considered to be the speed of light in ether space, his equations should be affected on the surface of the moving earth. But curiously, all electromagnetic phenomena as measured on the surface of the moving earth are independent of the movement of this planet. To dissolve this problem, Einstein (1905) assumes that Maxwell's equations are invariant to all measurers in steady motion which acts as the foundation of Special Relativity. This assumption of Einstein is possible when all four auxiliary Lorentz transforms are real. There is not a single proof that could properly justify Einstein's assumption. On the contrary it is now known that classical electrodynamics could easily explain all relativistic phenomena rationally.
\end{abstract}

Keywords: Auxiliary Lorentz Transforms; Real Lorentz Transforms; Classical Electrodynamics; Special Relativity

\section{Introduction}

Thomson's auxiliary co-ordinate transforms [1] along with the auxiliary time transform of Lorentz (1904) [2] are customarily called auxiliary Lorentz transforms. Auxiliary Lorentz Transforms are some mathematical constructs invented to solve electrodynamic problems classically. Those are made in such a way that Maxwell's equations are invariant in the auxiliary get-up too.

This paper deals with the nature of Auxiliary Lorentz transforms, their classical derivation and their relations with the real Lorentz Transforms extensively used in special relativity.

In Sections 2 and 3 of this paper, we have shown how Thomson (1889) invented auxiliary coordinate transforms to solve potential problems of steadily moving electromagnetic bodies electrostatically from the consideration of Maxwell [1] and how the auxiliary time transform of Lorentz could be used ingeniously to solve classically the radiating problems of a system of steadily moving radiating bodies in static format.

In Section 4, we have deduced the auxiliary time transform of Lorentz classically using auxiliary coordinate transforms of Thomson and Maxwell's equations directly.

In Sections 5 and 6, we have described the inherent reasons why Lorentz assumed that the co-ordinate transforms of Thomson are real and why Einstein assumed that all four auxiliary Lorentz Transforms are real.
In Sections 7 and 8, we have described the construction of the Special Relativity of Einstein that justifies that the auxiliary Lorentz Transforms are real and the consequences of such a justification.

Our goal is to show that 1) mathematics used in Special Relativity is exactly the same as that of Classical Electrodynamics and 2) the contention of the Special Relativity is not based on appropriate experimental evidence.

\section{Auxiliary Coordinate Transformation Equations of Thomson}

The potential $\left(\Phi_{0}\right)$ of a system of charges stationary in ether space is determined by the Poisson's equation

$$
\nabla^{2} \Phi_{0}=-\frac{\rho}{\varepsilon_{0}}, \Phi_{0}=\sum \frac{\rho \mathrm{d} \tau}{4 \pi \varepsilon_{0} r}
$$

But, the scalar potential $(\Phi)$ and the induced vector potential $\left(A^{*}\right)$ of this system of charges when steadily moves in the OX direction with a velocity $u$ in ether space are governed by D'Alembert's equation:

$$
\begin{gathered}
\square^{2} \Phi=-\frac{\rho}{\varepsilon_{0}} \\
\square^{2} A_{x}^{*}=-\frac{\rho u}{\varepsilon_{0} c^{2}}, A_{y}^{*}=0, A_{z}^{*}=0
\end{gathered}
$$

where $\rho$ is the charge density of the system, $\varepsilon_{0}$ is the permittivity and $\mu_{0}$ is the permeability of ether space 
such that $c=1 / \sqrt{\mu_{0} \varepsilon_{0}}$, and $x, y, z$ are the Cartesian coordinates introduced in ether space.

In such a situation when the system of charges steadily moves in ether space in the OX direction, the potentials at the point $(x, y, z)$ at the instant $t$ and those at the point $(x+u \mathrm{~d} t, y, z)$ at the instant $(t+\mathrm{d} t)$ in ether space will be the same. Therefore,

$$
\begin{gathered}
\Phi+\frac{\partial \Phi}{\partial t} \mathrm{~d} t+\frac{\partial \Phi}{\partial \mathrm{x}} u \mathrm{~d} t=\Phi \\
\frac{\partial \Phi}{\partial t}=-u \frac{\partial \Phi}{\partial x}, \quad \frac{\partial^{2} \Phi}{\partial t^{2}}=u^{2} \frac{\partial^{2} \Phi}{\partial x^{2}}
\end{gathered}
$$

Similarly,

$$
\frac{\partial A_{x}^{*}}{\partial t}=-u \frac{\partial A_{x}^{*}}{\partial x}, \frac{\partial^{2} A_{x}^{*}}{\partial t^{2}}=+u^{2} \frac{\partial^{2} A_{x}^{*}}{\partial x^{2}}
$$

Equations (5) and (6) are customarily called "steady state operators".

By the use of Equation (5), Equation (2) could be replaced by

$$
\left(1-u^{2} / c^{2}\right) \frac{\partial^{2} \Phi}{\partial x^{2}}+\frac{\partial^{2} \Phi}{\partial y^{2}}+\frac{\partial^{2} \Phi}{\partial z^{2}}=-\frac{\rho}{\varepsilon_{0}}
$$

and by the use of Equation (6), Equation (3) could be replaced by the Equations:

$$
\begin{gathered}
\left(1-u^{2} / c^{2}\right) \frac{\partial^{2} A_{x}^{*}}{\partial x^{2}}+\frac{\partial^{2} A_{x}^{*}}{\partial y^{2}}+\frac{\partial^{2} A_{x}^{*}}{\partial z^{2}}=-\frac{\rho u}{\varepsilon_{0} c^{2}} \\
A_{y}^{*}=0, A_{z}^{*}=0
\end{gathered}
$$

Comparing Equation (7) with Equation (8), we have,

$$
A_{x}^{*}=u \Phi / c^{2}
$$

Therefore, to determine $\boldsymbol{E}$ and $\boldsymbol{B}$, we are only to determine $\Phi$.

Now construct an auxiliary system (invented by Thomson) where the system of charges is stationary such that

$$
\begin{gathered}
x^{\prime}=\gamma x, y^{\prime}=y, z^{\prime}=z \\
\left(k=\sqrt{1-u^{2} / c^{2}}, \gamma=1 / k\right)
\end{gathered}
$$

and let charges remain the same in this system such that

$$
Q=\sum \rho \mathrm{d} \tau=\sum \rho^{\prime} \mathrm{d} \tau^{\prime}=\sum \rho^{\prime} \mathrm{d} x^{\prime} \mathrm{d} y^{\prime} \mathrm{d} z^{\prime}
$$

In this system Poisson's equation should be valid.

Therefore, in this auxiliary system, using Equations (11) and (12) we have, for auxiliary charge density

$$
\rho^{\prime}=\rho k
$$

The Poisson's Equation being still valid in this auxiliary system, we have,

$$
\nabla^{\prime} \Phi^{\prime}=-\rho^{\prime} / \varepsilon_{0}, \quad \Phi^{\prime}=\sum \frac{\rho^{\prime} \mathrm{d} \tau^{\prime}}{4 \pi \varepsilon_{0} r^{\prime}}
$$

$\Phi^{\prime}$ is the auxiliary potential of the auxiliary system and $r^{\prime}\left(r^{\prime 2}=x^{\prime 2}+y^{\prime 2}+z^{\prime 2}\right)$ is the auxiliary distance of the origin from the auxiliary position of the point where the field is to be determined.

The Equations (11) transforms Equation (7) to

$$
\nabla^{\prime 2} \Phi=-\rho / \varepsilon_{0}
$$
have,

Comparing Equation (15) with Equation (14), we

$$
\Phi=\gamma \Phi^{\prime}
$$

Thus we see that the potential of a moving system of charges is not connected to the potential of the same system at rest. That potential is $\gamma$ times of the potential of the stationary auxiliary system in which all the coordinates parallel to $\mathrm{OX}, \mathrm{OY}$ and $\mathrm{OZ}$ have been changed in the ratio determined by Equation (11). From Equation (16) we could very easily deduce many electrodynamic formula classically as given in [3].

For the potential $\Phi$ of a steadily moving point charge $Q$ we have from Equation (16),

$$
\Phi=\gamma \frac{Q}{4 \pi \varepsilon_{0} r^{\prime}}=\frac{Q}{4 \pi \varepsilon_{0} r\left(1-u^{2} \sin ^{2} \theta\right)^{1 / 2}}
$$

$\theta$ being the angle between the $\mathrm{OX}$ axis and the radius vector $r$ of the point $P$ where the potential is to be determined at the instant when the moving point charge is at the origin.

For the potential of a charge uniformly distributed over the surface of a sphere of radius $R$ moving with a velocity $u$ in ether space in OX direction,

$$
\begin{gathered}
\Phi=\gamma \Phi^{\prime}=\gamma \frac{Q}{8 \pi \varepsilon_{0} p} \ln \frac{\sqrt{p^{2}+l^{2}}+p}{\sqrt{p^{2}+l^{2}}-p} \\
p=a^{2}-b^{2}, \quad a=\gamma R, \quad b=R
\end{gathered}
$$

$l$ being the positive root of the equation

$$
\frac{x^{\prime 2}}{p^{2}+l}+\frac{y^{2}+z^{2}}{l}=1
$$

where in final calculation $x^{\prime}$ should be replaced by $\gamma x . \Phi^{\prime}$ here is the potential of a stationary ellipsoid of revolution in the auxiliary coordinate notation which could be constructed by the elongation of OX axis of the sphere by the factor $\gamma$ such that its semi-axis $=a$ is in the direction of motion and the equatorial radius $=b$.

\section{Auxiliary Time Transformation Equation of Lorentz}

The problem that Thomson addressed was to model the 
potentials for charges having a constant translational velocity in ether space. He solved this problem by transforming the D'Alembert's equation in an invariant form with Poisson's in the auxiliary system. Lorentz's problem was to model the radiation from steadily moving radiating bodies. He solved this problem by transforming Maxwell's equations for the moving radiating body to the same form for the auxiliary system, which correlates between the static and dynamic radiation states.

Suppose that a dipole stationary in ether space is emitting a train of plane monochromatic light wave of unit amplitude from the origin of a co-ordinate system $(x, y, z)$. The rays are chosen in $x-y$ plane, making an angle $\theta_{0}$ with the $\mathrm{OX}$ axis. Let us concentrate on the point $(x, y, z)$ such that $r=\left(x^{2}+y^{2}+z^{2}\right)^{1 / 2}$

The radiation equation should read,

$$
\square^{2} \boldsymbol{E}_{0}=0
$$

$\boldsymbol{E}_{0}$ being the radiating electric field at a point in ether space due to the system of charges inside the dipole stationary in ether space which implies a wave propagation in the form

$$
P_{0}=\cos \left[\omega_{0}\left(\frac{r}{c}-t\right)\right]
$$

Putting $\omega_{0}=2 \pi v_{0}$ and $r=x \cos \theta_{0}+y \sin \theta_{0}$ where $\omega_{0}$ is the radian frequency, $v_{0}$ is the frequency and $\lambda_{0}$ is the wave length of the propagating wave, an expression describing that propagation could be constructed from the propagation Equation (22) as

$$
P_{0}=\cos \left[2 \pi\left(\frac{x \cos \theta_{0}+y \sin \theta_{0}}{\lambda_{0}}-v_{0} t\right)\right]
$$

Now suppose that the dipole is moving with a velocity $u$ in the OX axis. What will be angle between the emitted ray and the direction of motion of the dipole?

When the dipole steadily moves, the electric and magnetic fields of the radiation fields of the dipole must change and thereby the ray if at all emitted must continue to move in a different direction.

We know that Heaviside's fields obey Maxwell's equations just like Coulomb's fields do [4]. Therefore, if the stationary dipole radiates, it must radiate, too, in steady motion [Verification could be found in Appendix A].

Therefore, when the above dipole steadily moves, the Maxwell's radiation equation should read

$$
\square^{2} \boldsymbol{E}=0
$$

$\boldsymbol{E}$ being the radiating electric field at a point in ether space due to the system of charges inside the dipole moving steadily in ether space.
The expression describing this propagation should read

$$
P=\cos \left[2 \pi\left(\frac{x \cos \theta+y \sin \theta}{\lambda}-v t\right)\right]
$$

The rays are too in $x-y$ plane, making an angle $\theta$ with the OX axis. In this case, the dynamic field $\boldsymbol{E}$ is not connected with the stationary field $\boldsymbol{E}_{0}$ by any direct relation. Therefore, $\theta$ could not be calculated in terms of $\theta_{0}$ by any direct equation. $\boldsymbol{E}$ is connected with $\boldsymbol{E}_{0}$ through the auxiliary field $\boldsymbol{E}^{\prime}$ constructed by the Thomson's auxiliary coordinate transformation equation. Therefore we are to calculate $\theta$ in terms of $\theta_{0}$ via $\theta^{\prime}$.

Now following Thomson, let us construct an auxiliary system defined by the following general coordinate transformation equations to study the potential problems of the steadily moving systems of electrodynamic bodies at $t>0$. These are as follows:

$$
x^{\prime}=\gamma(x-u t), y^{\prime}=y, z^{\prime}=z
$$

[The Equation (11) is used to study the situation at the time $t=0$ when the moving charge is at the origin of the frame fixed with the ether space. At other instants the changing electric and magnetic fields will look the same, although translated to the right by an amount ut. Therefore, in that case, the Equations (11) will be transformed to these equations of (26)].

Now, if the $\boldsymbol{E}$-field is transformed to the $\boldsymbol{E}^{\prime}$-field (the auxiliary field of Thomson) by the use of Thomson's auxiliary coordinate transformation equations (26), Maxwell's equations must not be valid in that system which one can easily verify by direct substitution. Thomson (1889) found that the auxiliary Equations (11) could transform the D'Alembert's equation of dynamic potential to the static Poisson's format in the auxiliary system which could facilitate the calculation of dynamic scalar and vector potentials correctly from the consideration of Maxwell. Lorentz (1904) found that if he would construct an auxiliary time transformation equation such that

$$
t^{\prime}=\gamma\left(t-u x / c^{2}\right)
$$

the dynamic radiation equation $\square^{2} \boldsymbol{E}=0$ will be transformed in the Thomson's Auxiliary system in the format

$$
\square^{\prime 2} \boldsymbol{E}^{\prime}=0
$$

(Vide the deduction in Section 4) and this, too, facilitate the radiation calculation of steadily moving radiating bodies correctly from the consideration of Maxwell as given below.

Equation (28) implies

$$
x^{\prime 2}+y^{\prime 2}+z^{\prime 2}=c^{2} t^{\prime 2}
$$

From Equation (28) we get,

$$
P^{\prime}=\cos \left[2 \pi\left(\frac{\mathrm{x}^{\prime} \cos \theta^{\prime}+y^{\prime} \sin \theta^{\prime}}{\lambda^{\prime}}-v^{\prime} t^{\prime}\right)\right]
$$


Now, Equation (25) and (30) are the same.

Relation between dynamic and auxiliary radiation quantities

Comparing Equation (25) and (30) we have,

$$
\begin{gathered}
\tan \theta=\frac{\sin \theta^{\prime} k}{\cos \theta^{\prime}+\beta}(\beta=u / c) \\
v=\gamma v^{\prime}\left(1+\beta \cos \theta^{\prime}\right)=\gamma c\left(1+\beta \cos \theta^{\prime}\right) / \lambda^{\prime}
\end{gathered}
$$

Relation between auxiliary and stationary radiation quantities

Now from the definition of the auxiliary system and the stationary system we have,

$$
\begin{gathered}
\sin \theta_{0}=\frac{y}{r}, \sin \theta^{\prime}=\frac{y^{\prime}}{r^{\prime}}, \cos \theta_{0}=\frac{x}{r}, \cos \theta^{\prime}=\frac{x^{\prime}}{r^{\prime}}, \\
r^{\prime 2}=x^{\prime 2}+y^{\prime 2}+z^{\prime 2}=\gamma^{2} r^{2}\left(1-\frac{u^{2}}{c^{2}} \sin ^{2} \theta_{0}\right)
\end{gathered}
$$

Relation between dynamic and stationary radiation quantities

Using Equations (31)-(33), we have for the angle $(\theta)$ of the ray with OX axis when the radiating system is steadily moving

$$
\begin{aligned}
\sin \theta^{\prime}=k \sin \theta_{0} / \alpha[\alpha & \left.=\left(1-u^{2} \sin ^{2} \theta_{0} / c^{2}\right)^{1 / 2}\right] \\
\cos \theta^{\prime} & =\cos \theta_{0} / \alpha
\end{aligned}
$$
(31),

Using Equations (34) and (35), we get from Equation

$$
\tan \theta=\frac{\sin \theta_{0} k^{2}}{\cos \theta_{0}+\alpha \beta}
$$

Similarly, for the calculation of frequency $(v)$ of the steadily moving radiating bodies we have from the last of Equation (33),

$$
\lambda^{\prime}=\alpha \gamma \lambda_{0}
$$

Using Equations (35) and (37), we have from Equation (32)

$$
v=\frac{v_{0}\left(\alpha+\beta \cos _{0}\right)}{\alpha^{2}}
$$

\section{Deduction of Lorentz' Auxiliary Time Transform}

When radiating body steadily moves in ether space Maxwell's Equation (24) implies

$$
x^{2}+y^{2}+z^{2}=c^{2} t^{2}
$$

To make the Maxwell's radiation equation invariant for the auxiliary system of Thomson, it is now required

$$
x^{\prime 2}+y^{\prime 2}+z^{\prime 2}=c^{2} t^{\prime 2}
$$

Solving Equations (39) and (40) using Equation (26), we get,

$$
t^{\prime}=\gamma\left(t-u x / c^{2}\right)
$$

This is the famous auxiliary time transform of Lorentz which along with the co-ordinate transforms of Thomson could recast Auxiliary radiation equation of Thomson in the format of Maxwell's radiation equation.

Thus we see that equations dealing with radiation from steadily moving radiating bodies could be readily deduced classically and correctly using the Auxiliary Lorentz transforms. Auxiliary Lorentz Transforms are classical. Therefore, results deduced by the use of those auxiliary equations are classical.

(An interesting feature of the Auxiliary Lorentz transforms is that the inverse of the Auxiliary Lorentz transforms are correct too. This means if

$$
x^{\prime}=\gamma(x-u t), y^{\prime}=y, z^{\prime}=z, t^{\prime}=\gamma\left(t-u x / c^{2}\right)
$$

are correct then the inverse equations i.e.,

$$
x=\gamma\left(x^{\prime}+u t^{\prime}\right), y=y^{\prime}, z=z^{\prime} t=\gamma\left(t^{\prime}+u x / c^{2}\right)
$$

are also correct which one could easily verify. This simple observation was used by A. Einstein in his Special Theory of Relativity.)

Auxiliary Lorentz transforms are the equations which express the relation between auxiliary $x^{\prime}, y^{\prime}, z^{\prime}, t^{\prime}$ and real $x, y, z, t$ in Classical Electrodynamics. The first three of the Auxiliary Lorentz Transforms could be used to study the scalar and vector potentials of any system of steadily moving charges and line current [3] but not to study the scalar and vector potentials of the similarly moving surface or volume currents. Auxiliary Lorentz Transforms conjointly are applicable only for point charge electrodynamics but not for large charge electrodynamics. These are applicable to study the cases when the system moves in ether space but the measurer is at rest. These could not be used to study the cases when the measurer moves but the system is at rest in ether space.

\section{Assumption of the Reality of the Thomson's Auxiliary System $\left(x^{\prime}, y^{\prime}, z^{\prime}\right)$ and Its Consequences}

To explain the null result of the Michelson-Morley Experiment, Fitzgerald in 1889 assumed that all bodies contract towards its direction of motion in ether space by an amount depending on the square of the ratio of their velocity to that of light.

According to Classical Electrodynamics when a charged sphere of radius $\mathrm{R}$ stationary in ether space moves in that space $(x, y, z)$ in a steady motion, the shape of the charged sphere remains the same, but the potential of this moving charged sphere should be $\gamma$ times of the auxiliary potential of an oblate ellipsoid of revolution with the 
axes $(\gamma R: R: R)$, in the auxiliary coordinate notations $\left(x^{\prime}, y^{\prime}, z^{\prime}\right)$ to be finally replaced by real coordinate notations $(x, y, z)[\gamma R$ being in the direction of motion, vide Equation (18)]. This means that relative to the stationary auxiliary system, the moving electromagnetic system is contracted with the $k$ factor towards its direction of motion.

All measurements we use in electrodynamics are performed on the surface of the moving earth and these are real. Therefore the dynamic system in electrodynamics is real. But the stationary ether system is illusive.

To match electrodynamic principles with Fitzgerald's assumption, Lorentz assumed with novelty that Auxiliary coordinate transforms of Thomson are real (effective/ true). This means that when an electrodynamic body (as well as any mechanical body) steadily moves in ether space, it contracts with $k$ factor towards its direction of motion and the auxiliary quantities of the stationary auxiliary system too, act as real quantities as in the real stationary system. This somehow explains the null result of the Michelson-Morley Experiment with some distortion and obscurity of Classical Electrodynamics.

We measure electrodynamic quantities on the surface of the moving earth and these quantities are real. Electrodynamic quantities relating to the electromagnetic system at rest in ether space is illusive. Lorentz assumed the effectiveness (reality) of the Thomson's Auxiliary system which lessened the importance of ether space in his electrodynamics.

According to Lorentz' contraction hypothesis the aforesaid spherical charge changes to an contracted ellipsoid $(k R: R: R)$ while in steady motion and its scalar potential $(\Phi)$ becomes $\gamma$ times of the scalar potential of a stationary charged sphere of radius $R$ in the auxiliary notations to be finally transformed to dynamic notation notations as given in Equation (17).

The calculation and interpretation on the effects of a moving point charge are same to both Classical Electrodynamics and Lorentz' contraction hypothesis; as the geometry of the point charge remains the same in the auxiliary system. But the calculation for steadily moving charged big bodies are different as per these two different interpretations.

Lorentz always advocated for his moving contracted ellipsoidal electron against spherical electron of Abraham. He tried to establish that his electron had advantage over Abraham's which was his bias.

Lorentz considered his time $t^{\prime}$ as local time.

\section{Assumption of the Reality of Lorentz' Auxiliary Time $t^{\prime}$}

In the Maxwell's theory, if $c$ is considered to be the speed of light in ether space, Maxwell's equations are then valid in ether space where the earth is obviously moving with an appreciable velocity. Therefore, the Maxwell's equations should be affected on the surface of the moving earth. But curiously, all electromagnetic phenomena as measured on the surface of the moving earth are independent of the movement of this planet.

To dissolve this problem, Einstein (1905) assumes that Maxwell's equations are invariant to all measurers in steady relative motion which abolishes absolute space and acts as the foundation of Special Relativity. This assumption of Einstein is possible when all four auxiliary quantities in Lorentz transforms (which are deduced to make Maxwell's equation invariant in the auxiliary system as shown in Section 4) are real to those measurers, which abolishes the polemical preferred frame wherein physical laws were considered to preserve their simplest forms. In this interpretation, auxiliary time $t^{\prime}$ is real too along with auxiliary coordinates.

Then Einstein somehow tried to justify the reality of those auxiliary quantities and their reciprocity in the following way.

\section{Construction of Special Relativity}

Lorentz transforms could be deduced algebraically from many sets of arbitrary equations. Einstein [5] chose such two sets of equations, the first set viz.,

1) $x^{\prime}=\gamma(x-u t), y^{\prime}=y, z^{\prime}=z$

2) $x=\gamma\left(x^{\prime}+u t^{\prime}\right), y=y^{\prime}, z=z^{\prime}$

[From the Standard \& inverse Lorentz transforms Equations (42) and (43)] $\gamma$ being unknown, and the second set, viz.,

1) $x^{2}+y^{2}+z^{2}=c^{2} t^{2}$

2) $x^{\prime 2}+y^{\prime 2}+z^{\prime 2}=c^{2} t^{\prime 2}$

[From real dynamic radiation Equation (24) and its auxiliary (28)] from which Lorentz Transforms could be recovered.

Now, Einstein assumed that both the sets of above equations are real (which means that auxiliary state in classical electrodynamics is itself real) and therefore, Lorentz Transforms deduced from those two sets of equations are themselves real. The reality of the Lorentz transforms to the measurers in steady relative motion implies that distance and time are relative.

Einstein, however, liked to describe physics in terms of principles (assumptions). He stated his first set of assumption with certainty: the laws of physics are the same in all inertial frames. No preferred inertial frame exists. This is the First Principle which means that his first set of equations is real to measurers in steady relative motion. The first principle is customarily called "the principle of relativity".

Similarly, he stated his second set of assumption with 
the same certainty: the speed of light is the same " $c$ " in all inertial frames. That means that his second set of equations is real to measurers in steady relative motion. The second principle is customarily called the "the principle of the constancy of the speed of light".

Now, if those two principles are real, then Lorentz Transforms are real too. This conclusion constitutes special relativity theory which contradicts Classical Physics.

In such an interpretation, all the auxiliary equations as given in Sections 2, 4-6 are real. In Section 3, instead of Classical Equations (36) and (38), the auxiliary Equations (31) and (32) are accepted as correct calculations as per Special Relativity.

Real Lorentz transforms as per Einstein are the equations which express the relation between real $x^{\prime}, y^{\prime}, z^{\prime}, t^{\prime}$ and real $x, y, z, t$. According to him the equations are valid universally in any case of relative steady motion.

\section{Consequences of the Assumptions of the Reality of Lorentz' Auxiliary System}

In his explanation, A. Einstein has tried to remove the question on the real contraction of moving electrons advocated by Lorentz. According to this interpretation, the shape of the electron remains the same whereas different measurers with different relative velocities should measure the shape of the electron differently.

To Einstein all auxiliary Lorentz transforms including the auxiliary time transformation equation are real, absolutely exact and applicable to every field of physics. Thus when auxiliary Lorentz transforms are transcended as reality to all realm of physics, length contracts, inertial mass varies or converts into energy and more interestingly time dilates with velocity!

It has been proved with certainty that the speed of light is the same $c$ on the surface of the moving earth which possesses appreciable gravity on its surface. However, there is not a single experiment to show that the speed of light is the same $\mathrm{c}$ in an inertial frame (with very negligible or no gravity) steadily moving in ether space. Therefore, the second principle does not have any physical foundation.

Similarly, Maxwell's radiation equation is seen to be invariant on the moving earth but it is not proved by any experiment that the equation preserves its invariant form in an inertial frame with no or negligible gravity steadily moving in ether space. Therefore, the first principle, too, lacks in scientific rigor for which the theory boast.

In the domain of Classical electrodynamics, Auxiliary Lorentz transforms are actually the relation of co-ordinates and time between the imaginary auxiliary state of Classical Electrodynamics and real dynamic state. Relativists make an effort to justify the reality from the assumption that auxiliary state of Classical Electrodynam- ics itself is real.

But from the consideration of Classical Electrodynamics, the reality of such assumption has not been proved by any experiments.

For point charges both the calculations are the same. But for large charge electrodynamics both the calculations markedly differ. So, the efforts of the relativists could be successful if they cite the results of large charge experiments conforming only to their calculations which they avoid.

To justify reality of auxiliary state beyond Classical electrodynamics, relativists takes the advantage of the situation that for bodies like planets and satellites,

$\sqrt{1-u^{2} / c} \rightarrow 1$ and so they concludes that both the results will be same, for which they are not eager to verify their conclusion by experiment.

There is not a single experiment to prove that moving bodies really contract with the same factor $k$.

As per classical electrodynamics, when a radiating source having frequency $v$ moves away in ether space with a velocity $u$ from a stationary receiver with an electromagnetic clock, the frequency of the source will decrease to $v k$ ([3], Equation (80)) and the stationary receiver will measure the frequency of the source as $v^{\prime}=v k /(1-u / c) ;$ but in case the source is at rest and the receiver similarly moves in opposite direction, the frequency of the source remains the same, the receiver's one second will increase to $\gamma$ seconds ([3], Equation (83)) and the moving receiver will measure the frequency as $v^{\prime}=v(1+u / c) \gamma$, both of which are the same. But if the receiver measures the frequency with a mechanical clock such that the ticking of the clock does not change with velocity, the moving receiver will measure the frequency as $v^{\prime}=v(1+u / c)$. Therefore, Ives-Stilwell experiment could not be used to uphold relativistic space-time as well as equivalence principle. The issue could only be settled by the use of mechanical clocks which do not change ticking with velocity.

Reality of the auxiliary quantities in the Auxiliary Lorentz transforms has not been proved by the relativists.

Therefore, till this day, in the true sense, the theory of relativity could not claim its validity experimentally. The theory is still now a proposition only.

\section{Conclusions}

This paper concludes that the Special Relativity Theory stands only on the proposition that the auxiliary Lorentz transformation equations of Classical Electrodynamics are real.

But the reality of those equations has not been proved by any experiments. All electrodynamic phenomena could easily and rationally be explained from classical physics only replacing ether space by free space where 
there is negligible or no gravity to disturb electromagnetic fields which are real and subject to gravitation [3].

\section{Acknowledgements}

I thank Alak Bandyopadhyay for his kind help to prepare the manuscript.

\section{REFERENCES}

[1] J. J. Thomson, "On the Magnetic Effects Produced by Motion in the Electric Field," Philosophical Magazine, Vol. 28, No. 170, 1889, pp. 1-14. doi: $10.1080 / 14786448908619821$

\section{Appendix A}

It has been shown in [4] that Heaviside's fields obey Maxwell's equations just like Coulomb's fields do. Therefore, if a stationary dipole radiates in ether space, it will also radiate while moving in ethers pace at constant translational velocity which we may verify as follows:

Let us consider an oscillating dipole whose centre is stationary in ether space. Let us further consider that the direction of oscillation is OZ.

The components of the electric field $\left(\boldsymbol{E}_{0}\right)$ and the magnetic field $\left(\boldsymbol{B}_{0}\right)$ due to this dipole are as follows:

$$
\begin{gathered}
\left(\boldsymbol{E}_{0}\right)_{x}=-A_{0} \frac{x z}{r^{3}},\left(\boldsymbol{E}_{0}\right)_{y}=-A_{0} \frac{z y}{r^{3}},\left(\boldsymbol{E}_{0}\right)_{z}=A_{0} \frac{x^{2}+y^{2}}{r^{2}} \\
\left(\boldsymbol{B}_{0}\right)_{x}=\frac{-A_{0}}{c} \frac{y}{r},\left(\boldsymbol{B}_{0}\right)_{y}=\frac{A_{0}}{c} \frac{x}{r},\left(\boldsymbol{B}_{0}\right)_{z}=0
\end{gathered}
$$

As expected $\boldsymbol{E}_{0}=c \boldsymbol{B}_{0}$ and $\boldsymbol{E}_{0} \cdot \boldsymbol{B}_{0}=0$ which satisfies the radiation condition.

Now suppose that the dipole is steadily moving in the OX direction.

Then the components of the auxiliary fields are as follows:
[2] H. A. Lorentz, "Electromagnetic Phenomena in a System Moving with Any Velocity Smaller than That of Light," Proceedings of the Royal Netherlands Academy of Arts and Sciences, Vol. 6, 1904, pp. 809-831.

[3] S. Hajra, "Classical Interpretations of Relativistic Phenomena," Journal of Modern Physics, Vol. 3, No. 2, 2012, pp. 187-199. doi:10.4236/jmp.2012.32026

[4] P. Lorrain and D. Corson, "Electromagnetic Fields and Waves," 2nd Edition, CBS Publishers and Distributors, Delhi, 1986, pp. 271-276.

[5] A. Einstein, "On the Electrodynamics of Moving Bodies," Annalen der Physik, Vol. 17, 1905, pp. 891-921. doi:10.1002/andp.19053221004

$$
\begin{gathered}
\boldsymbol{E}_{x}^{\prime}=-A_{0} \frac{x^{\prime} z^{\prime}}{r^{\prime 3}}, \boldsymbol{E}_{y}^{\prime}=-A_{0} \frac{z^{\prime} y^{\prime}}{r^{\prime 3}}, \boldsymbol{E}_{z}^{\prime}=A_{0} \frac{x^{\prime 2}+y^{\prime 2}}{r^{\prime 2}} ; \\
\boldsymbol{B}_{x}^{\prime}=\frac{-A_{0}}{c} \frac{y^{\prime}}{r^{\prime}}, \boldsymbol{B}_{y}^{\prime}=\frac{A_{0}}{c} \frac{x^{\prime}}{r^{\prime}}, \boldsymbol{B}_{z}^{\prime}=0 .
\end{gathered}
$$

Following the Equations (27) and (28) of [3], the components of the dynamic fields are as follows [first of the Equations (27) in [3] should be corrected to $\boldsymbol{E}_{x}=\boldsymbol{E}_{x}^{\prime}$ :

$$
\begin{gathered}
\boldsymbol{E}_{x}=-A_{0} \frac{x^{\prime} z^{\prime}}{r^{\prime 3}}, \boldsymbol{E}_{y}=-\gamma A_{0} \frac{z^{\prime} y^{\prime}}{r^{\prime 3}}, \\
\boldsymbol{E}_{z}=\gamma A_{0}\left[\frac{x^{\prime 2}+y^{\prime 2}}{r^{\prime 2}}-\beta \frac{x^{\prime}}{r^{\prime}}\right] ; \\
\boldsymbol{B}_{x}=\frac{-A_{0}}{c} \frac{y^{\prime}}{r^{\prime}}, \boldsymbol{B}_{y}=\gamma A_{0}\left[\frac{x^{\prime}}{c r^{\prime}}-\frac{u}{c^{2}} \frac{x^{\prime 2}+y^{\prime 2}}{r^{\prime 2}}\right], \\
\boldsymbol{B}_{z}=-\gamma A_{0} \frac{u}{c^{2}} \frac{y^{\prime} z^{\prime}}{r^{\prime 2}}\left(\beta=\frac{u}{c}\right) .
\end{gathered}
$$

It is trivial to show that $\boldsymbol{E}=c \boldsymbol{B}$ and $\boldsymbol{E} \cdot \boldsymbol{B}=0$ which satisfies the radiation condition which is in conformity with the proposition. 